\title{
Phytochrome diversification in cyanobacteria and eukaryotic algae
}

Nathan C. Rockwell and J. Clark Lagarias*

Department of Molecular and Cellular Biology, 31 Briggs Hall, One Shields Avenue, University of California, Davis, CA 95616.

*Author for correspondence: J. Clark Lagarias, Tel: 530-752-1865, email jclagarias@ucdavis.edu 


\begin{abstract}
(100-120 words max)
Phytochromes control almost every aspect of plant biology, including germination, growth, development, and flowering, in response to red and far-red light. These photoreceptors thus hold considerable promise for engineering crop plant responses to light. Recently, structural research has shed new light on how phytochromes work. Genomic and transcriptomic studies have improved our understanding of phytochrome loss, retention, and diversification during evolution. We are also beginning to understand phytochrome function in cyanobacteria and eukaryotic algae.
\end{abstract}




\section{Introduction}

To feed an expanding population with limited arable land, we must improve the

performance of crop plants [1]. The plant shade avoidance response can be a limiting factor in high-density crop plantings and is controlled by phytochrome photoreceptors [2]. Plant phytochromes act as master regulators of growth and development in response to light (photomorphogenesis), controlling thousands of genes [3]. The phytochrome superfamily is widespread, and structural studies on bacterial phytochromes have provided great insights into these proteins. In the last three years, research has shed new light on how phytochromes work, on the origins of plant phytochromes, on the distribution of phytochromes in eukaryotic algae, and on the roles these proteins play in cyanobacterial and algal photobiology.

\section{Phytochrome structure and signaling mechanisms}

The phytochrome superfamily comprises photoreceptors having a conserved GAF domain that binds a linear tetrapyrrole (bilin) chromophore (Fig. 1). This GAF domain can be isolated in cyanobacteriochromes (CBCRs) or can be part of a larger photosensory core module (PCM) in phytochromes [4]. The phytochrome PCM "input" is typically Nterminal and can be fused to different C-terminal signaling "output" domains such as histidine kinases. Some cyanobacterial phytochromes have a 2-domain PCM comprising an N-terminal GAF domain and a C-terminal PHY domain, whereas more widely distributed phytochromes have an N-terminal PAS domain in a larger PAS-GAF-PHY PCM (Fig. 1). Crystal structures have established the presence of an unusual knotted architecture at the PAS-GAF interface and of a conserved, flexible loop (or tongue) in the 
PHY domain that contacts the chromophore-binding pocket in the GAF domain $[5,6]$. Light absorption by the bilin chromophore triggers photoisomerization of the 15,16 double bond of the bilin chromophore [7,8], flipping the bilin D-ring relative to the rest of the chromophore and triggering a rearrangement of protein-chromophore interactions.

The two chromophore configurations thus have different spectral properties. In plants, the $15 Z$ configuration $\left(\mathrm{P}_{\mathrm{r}}\right)$ is autocatalytically generated upon assembly with bilin, is stable in darkness, and absorbs red light, whereas the $15 E$ configuration $\left(\mathrm{P}_{\mathrm{fr}}\right)$ is generated by light absorption, is metastable, and absorbs far-red light (Fig. 2). Similar red/far-red photocycles are known in a range of phytochromes from algae, cyanobacteria, other bacteria, and fungi [4,9]. Structural and biochemical studies of bacterial phytochromes have recently provided new insights into the mechanisms coupling photoconversion to changes in the signaling state of the output domain [10-14]. The tongue of the PHY

domain undergoes a structural rearrangement upon photoconversion that leads to a rotation of the C-terminal output domain relative to the PCM. This rotation can alter catalytic activity and/or dimerization affinity, thereby changing the signaling state of phytochrome. The similar structures of bacterial and plant phytochromes make it likely that this paradigm will hold true for eukaryotic phytochromes as well [15].

\section{The contested origins of plant phytochrome}

The discovery of phytochromes in cyanobacteria led to the hypothesis that a cyanobacterial photosensory gene was transferred to the host nucleus during plant evolution [16]. A recent phylogenetic analysis has provided some support for this hypothesis [17], with a proposed fusion of a cyanobacterial PCM to an existing host 
histidine kinase. Other analyses do not agree with this conclusion $[18,19]$. Instead, these studies are consistent with descent of plant phytochrome from an ancestral host gene, with loss of cyanobacterial photosensors during chloroplast evolution. These studies incorporated more extensive sampling of phytochromes from distantly related stramenopile (or heterokont) algae, reducing the risk of artifacts caused by long branch attraction. The presence of phytochromes in fungi and stramenopiles is consistent with the presence of an ancient eukaryotic phytochrome that has been repeatedly lost in evolution.

However, an additional complication is suggested by a recent study of ferredoxindependent bilin reductases (FDBRs), the enzymes carrying out the last step in bilin biosynthesis in plants, cyanobacteria, and eukaryotic algae [20]. In this study, plant HY2 enzymes are sister to extant cyanobacterial PebB sequences rather than derived from them, despite the cyanobacterial origin of all FDBRs. This result implies that genetic drift in cyanobacterial sequences after establishment of primary endosymbiosis is sufficient to mask ancestral relationships. Were such a case to apply in phytochromes, an ancient cyanobacterial PCM could be a common ancestor for extant plant and cyanobacterial phytochromes but might not be identified as such if compared to extant cyanobacterial phytochromes.

\section{Loss and retention of phytochrome in photosynthetic organisms}

Our inability to resolve the origins of plant phytochromes echoes similar difficulties in unraveling the origins of eukaryotic photosynthesis $[21,22]$. This situation is exacerbated by repeated examples of phytochrome loss and radiation in evolution of different 
lineages. For example, cyanobacteria have at least four distinct phytochrome lineages: knotless GAF-PHY phytochromes, knotted PAS-GAF-PHY phytochromes that are similar to plant phytochromes, knotted PAS-GAF-PHY phytochromes that are similar to phytochromes from other bacteria, and unusual phytochromes able to sense light at much shorter wavelengths $[4,23]$. Many cyanobacteria also contain CBCRs, which are distant phytochrome relatives that require only a bilin-binding GAF domain to generate more diverse spectral responses [4,23-26]. Phytochromes have also been lost in some cyanobacteria. For example, Thermosynechococcus elongatus contains only CBCRs, whereas Prochlorococcus strains and closely related Cyanobium and Synechococcus strains have lost all phytochromes and CBCRs [24,27].

Recent genomic and transcriptomic studies allow an almost complete assessment of phytochrome distribution in photosynthetic eukaryotes [28,29]. There are two known examples of primary endosymbiosis of a cyanobacterium for carbon fixation (Fig. 3). In the most recent event, rhizarian amoebae of the genus Paulinella acquired photosynthesis via endosymbiosis of a Cyanobium or Synechococcus strain [30,31]. Genome sequencing for one such organism, Paulinella chromatophora, did not detect phytochrome sequences (Fig. 3). The more ancient endosymbiosis has been proposed to give rise to a single lineage (Archaeplastida) comprising glaucophyte and rhodophyte algae and Viridiplantae (green algae and plants), although this is not universally recovered [21,22]. Phytochromes are apparently ubiquitous in glaucophytes but absent in extant rhodophytes (Fig. 3). Viridiplantae comprises a prasinophyte/chlorophyte lineage, in which phytochromes are sometimes present but have been repeatedly lost, and a streptophyte lineage, which includes land plants and in which phytochromes are ubiquitous $[18,28,29]$. Glaucophyte 
and Viridiplantae phytochromes constitute a single lineage, establishing that phytochromes were likely present in either the cyanobacterium or the host cell at the time of endosymbiosis [18,19].

Paulinella chromatophora and Archaeplastida have arisen via primary endosymbioses of free-living cyanobacteria. However, subsequent endosymbiotic events have given rise to a wide range of algae (Fig. 3). Secondary endosymbiosis of rhodophyte algae led to haptophytes, cryptophytes, photosynthetic stramenopiles (Ochrophyta), and photosynthetic alveolates (chromerids and dinoflagellates: Fig. 3). Secondary endosymbiosis of prasinophyte or chlorophyte algae has given rise to chlorarachniophyte algae and photosynthetic euglenids. At least two dinoflagellate lineages have subsequently carried out tertiary endosymbiosis of haptophyte algae (Kareniaceae) or diatoms (dinotoms, Fig. 3).

Phytochrome is not present in all such complex algae. Current data for euglenids are not conclusive [28,32,33], but phytochrome is absent in haptophytes, chromerids, and chlorarachniophytes (Fig. 3). Phytochrome is present in some but not all photosynthetic stramenopiles and has radiated into a small gene family in complex phaeophyte algae (kelps) [9,34,35]. Most dinoflagellates lack phytochromes, but recent transcriptomic studies on the dinotom Durinskia baltica [36] have shown that its diatom symbiont retains a phytochrome gene (Fig. 3). Phytochromes are ubiquitous in photosynthetic cryptophytes but absent in transcriptomes for basal, nonphotosynthetic members of the Cryptista (goniomonads and Palpitomonas bilix) [18,28]. Cryptophyte PCM sequences are part of a single lineage also including glaucophyte and Viridiplantae phytochromes, but the evolutionary history of cryptophyte phytochromes remains enigmatic [18,19]. 
Overall, phytochrome is universally conserved in neither cyanobacteria nor photosynthetic eukaryotes and hence cannot have globally conserved, essential functions in oxygenic photosynthetic organisms.

\section{Phytochrome function in cyanobacteria}

Some phytochrome functions in cyanobacteria may have been taken over by CBCRs, which are known to control phototaxis and complementary chromatic acclimation [25,26,37]. The knotless phytochrome Cph2 from Synechocystis sp. PCC 6803, which is involved in phototactic responses to blue light, has both a phytochrome PCM and a CBCR domain which responds to blue light [38]. However, cyanobacterial phytochromes can also function independently of CBCRs. The knotless phytochrome RfpA is essential for far-red-light photoacclimation (FaRLiP), a physiological response allowing growth on far-red light by remodeling the photosynthetic apparatus and inducing synthesis of redshifted chlorophylls [39-42]. RfpA is part of a large cluster of FaRLiP genes that have apparently spread by lateral transfer $[43,44]$. These studies thus demonstrate that cyanobacterial phytochromes can provide dedicated photobiological functions permitting optimization of cellular metabolism for a given light environment.

\section{Phytochrome function in eukaryotic algae}

Eukaryotic algal phytochromes all have a PAS-GAF-PHY PCM, unlike the more diverse cyanobacterial phytochromes (Fig. 1). However, this stereotyped PCM architecture is not accompanied by a conserved red/far-red photocycle. Phytochromes from land plants and streptophyte algae exhibit such photocycles, but photocycles from other algae exhibit unexpected diversity [9]. Phytochromes from prasinophyte algae can exhibit yellow/far- 
red or orange/far-red photocycles as well as red/far-red ones. Phytochromes from glaucophyte algae exhibit blue/far-red or red/blue photocycles, and phytochromes from stramenopiles can exhibit red/far-red or far-red/green photocycles. There is a rough correlation between the peak absorption of the dark-stable state of a given algal phytochrome and the depth inhabited by its host alga, consistent with the wavelengthdependent properties of light attenuation by water $[9,45]$. However, further work is needed to establish any functional significance of this trend.

Eukaryotic algal phytochromes also have diverse C-terminal architectures $[18,19,46]$. Many such proteins have C-terminal histidine kinases or related domains, but the presence of one or more response regulator receiver (REC) domains is variable. A detailed phylogenetic analysis demonstrated that canonical plant phytochromes arose from streptophyte algal ancestors having REC domains. Further diversity is seen in some phytochromes from cryptophytes and the moss Ceratodon purpureus, which have Cterminal Ser/Thr kinases [19]. Cryptophyte examples, termed Phytochrome Eukaryotic Kinases (PEKs) [18,19], can also have a C-terminal RING domain (Fig. 1) and hence may be implicated in ubiquitination and protein turnover. A Ser/Thr kinase output domain is also found in neochrome photoreceptors, which arose in streptophyte algae via fusion of an N-terminal red/far-red phytochrome to a flavin-based phototropin photoreceptor [46]. Some prasinophyte phytochromes also have candidate C-terminal adenylate cyclase domains (Fig. 1). This diversity implies that algal phytochromes may have functions not seen in higher plants.

Our understanding of phytochrome function in algae is in its infancy. Reports of far-red photobiology in euglenids are consistent with the possible presence of phytochromes in 
Eutreptiella gymnastica strain CCMP1594 [28,47]. In streptophyte algae and some nonvascular plants, neochromes function in the cytosol to mediate changes in plastid orientation and/or directional growth in response to light [48]. Higher plant phytochromes function in both the cytosol and the nucleus, with candidate interacting proteins identified in both compartments [49]. Light-dependent nuclear import of plant phytochromes is a critical step in inducing photomorphogenesis [50]. The light-dependent accumulation of phytochrome in the nucleus of the prasinophyte alga Micromonas pusilla thus implicates light-dependent nuclear localization as a conserved mechanism of phytochrome function throughout the Viridiplantae [18]. A red/far-red phytochrome regulates gene expression in response to the levels of far-red light in the diatom Phaeodactylum tricornutum [35], and the presence of apparent orthologs in many diatoms implicates similar functions in such organisms as well. Hopefully, these promising studies will lead to further insights in the near future.

\section{Conclusions and future prospects}

Phytochromes provide a wide range of organisms with the ability to tune their metabolism in response to the light environment via processes such as shade avoidance and FaRLiP. Recent studies have demonstrated that plant phytochromes evolved from algal ancestors of uncertain origin. Both algal and cyanobacterial phytochromes are more spectrally diverse than land plant phytochromes, perhaps reflecting more diverse light

environments in water than on land. Light-dependent nuclear localization of phytochromes is conserved in streptophytes and prasinophytes, and the future will continue to shed light on these fascinating photoreceptors. 


\section{Acknowledgements}

This work was supported by NIH grant R01 GM068552 (to J. C. L.) and by a grant from the Chemical Sciences, Geosciences, and Biosciences Division, Office of Basic Energy Sciences, Office of Science, United States Department of Energy (DOE DE-FG0209ER16117 to J.C.L).

\section{References}

1. Ort DR, Merchant SS, Alric J, Barkan A, Blankenship RE, Bock R, Croce R, Hanson MR, Hibberd JM, Long SP, et al.: Redesigning photosynthesis to sustainably meet global food and bioenergy demand. Proc. Natl. Acad. Sci. USA 2015, 112:8529-8536.

2. Casal JJ: Photoreceptor signaling networks in plant responses to shade. Ann. Rev. Plant Biol. 2013, 64:403-427.

3. Franklin KA, Quail PH: Phytochrome functions in Arabidopsis development. J. Exp. Bot. 2010, 61:11-24.

4. Rockwell NC, Lagarias JC: A brief history of phytochromes. ChemPhysChem 2010, 11:1172-1180.

5. Hughes J: Phytochrome three-dimensional structures and functions. Biochem. Soc. Trans. 2010, 38:710-716.

6. Wagner JR, Brunzelle JS, Forest KT, Vierstra RD: A light-sensing knot revealed by the structure of the chromophore binding domain of phytochrome. Nature 2005, 438:325-331.

7. Song C, Psakis G, Lang C, Mailliet J, Gartner W, Hughes J, Matysik J: Two ground state isoforms and a chromophore D-ring photoflip triggering extensive intramolecular changes in a canonical phytochrome. Proc. Natl. Acad. Sci. USA 2011, 108:3842-3847.

8. Yang X, Ren Z, Kuk J, Moffat K: Temperature-scan cryocrystallography reveals reaction intermediates in bacteriophytochrome. Nature 2011, 479:428-432.

9. Rockwell NC, Duanmu D, Martin SS, Bachy C, Price DC, Bhattacharya D, Worden AZ, Lagarias JC: Eukaryotic algal phytochromes span the visible spectrum. Proc. Natl. Acad. Sci. USA 2014, 111:3871-3876.

10. Takala H, Bjorling A, Berntsson O, Lehtivuori H, Niebling S, Hoernke M, Kosheleva I, Henning R, Menzel A, Ihalainen JA, et al.: Signal amplification and transduction in phytochrome photosensors. Nature 2014, 509:245-248.

11. Bjorling A, Berntsson O, Takala H, Gallagher KD, Patel H, Gustavsson E, St Peter R, Duong P, Nugent A, Zhang F, et al.: Ubiquitous Structural Signaling in Bacterial Phytochromes. J. Phys. Chem. Lett. 2015, 6:3379-3383. 
12. Takala H, Bjorling A, Linna $M$, Westenhoff $S$, Ihalainen JA: Light-induced Changes in the Dimerization Interface of Bacteriophytochromes. J. Biol. Chem. 2015, 290:16383-16392.

13. Bjorling A, Berntsson O, Lehtivuori H, Takala H, Hughes AJ, Panman M, Hoernke M, Niebling S, Henry L, Henning R, et al.: Structural photoactivation of a fulllength bacterial phytochrome. Sci. $A d v$. 2016, 2:e1600920.

14. Burgie ES, Zhang J, Vierstra RD: Crystal Structure of Deinococcus Phytochrome in the Photoactivated State Reveals a Cascade of Structural Rearrangements during Photoconversion. Structure 2016, 24:448-457.

15. Burgie ES, Bussell AN, Walker JM, Dubiel K, Vierstra RD: Crystal structure of the photosensing module from a red/far-red light-absorbing plant phytochrome. Proc. Natl. Acad. Sci. USA 2014, 111:10179-10184.

16. Yeh K-C, Wu S-H, Murphy JT, Lagarias JC: A cyanobacterial phytochrome twocomponent light sensory system. Science 1997, 277:1505-1508.

17. Buchberger T, Lamparter T: Streptophyte phytochromes exhibit an $\mathbf{N}$-terminus of cyanobacterial origin and a $\mathbf{C}$-terminus of proteobacterial origin. $B M C$ Res. Notes 2015, 8:144.

18. Duanmu D, Bachy C, Sudek S, Wong CH, Jimenez V, Rockwell NC, Martin SS, Ngan CY, Reistetter EN, van Baren MJ, et al.: Marine algae and land plants share conserved phytochrome signaling systems. Proc. Natl. Acad. Sci. USA 2014, 111:15827-15832.

19. Li FW, Melkonian M, Rothfels CJ, Villarreal JC, Stevenson DW, Graham SW, Wong GK, Pryer KM, Mathews S: Phytochrome diversity in green plants and the origin of canonical plant phytochromes. Nat. Commun. 2015, 6:7852.

20. Rockwell NC, Martin SS, Li FW, Mathews S, Lagarias JC: The phycocyanobilin chromophore of streptophyte algal phytochromes is synthesized by HY2. New Phytol. 2017, in press.

21. Price DC, Chan CX, Yoon HS, Yang EC, Qiu H, Weber AP, Schwacke R, Gross J, Blouin NA, Lane C, et al.: Cyanophora paradoxa genome elucidates origin of photosynthesis in algae and plants. Science 2012, 335:843-847.

22. Burki F, Kaplan M, Tikhonenkov DV, Zlatogursky V, Minh BQ, Radaykina LV, Smirnov A, Mylnikov AP, Keeling PJ: Untangling the early diversification of eukaryotes: a phylogenomic study of the evolutionary origins of Centrohelida, Haptophyta and Cryptista. Proc. Royal Soc. B Biol. Sci. 2016, 283.

23. Rockwell NC, Martin SS, Feoktistova K, Lagarias JC: Diverse two-cysteine photocycles in phytochromes and cyanobacteriochromes. Proc. Natl. Acad. Sci. USA 2011, 108:11854-11859.

24. Ikeuchi M, Ishizuka $\mathrm{T}$ : Cyanobacteriochromes: a new superfamily of tetrapyrrole-binding photoreceptors in cyanobacteria. Photochem. Photobiol. Sci. 2008, 7:1159-1167.

25. Song JY, Cho HS, Cho JI, Jeon JS, Lagarias JC, Park YI: Near-UV cyanobacteriochrome signaling system elicits negative phototaxis in the cyanobacterium Synechocystis sp. PCC 6803. Proc. Natl. Acad. Sci. USA 2011, 108:10780-10785. 
26. Hirose Y, Rockwell NC, Nishiyama K, Narikawa R, Ukaji Y, Inomata K, Lagarias JC, Ikeuchi M: Green/red cyanobacteriochromes regulate complementary chromatic acclimation via a protochromic photocycle. Proc. Natl. Acad. Sci. USA 2013, 110:4974-4979.

27. Bibby TS, Mary I, Nield J, Partensky F, Barber J: Low-light-adapted Prochlorococcus species possess specific antennae for each photosystem. Nature 2003, 424:1051-1054.

28. Keeling PJ, Burki F, Wilcox HM, Allam B, Allen EE, Amaral-Zettler LA, Armbrust EV, Archibald JM, Bharti AK, Bell CJ, et al.: The Marine Microbial Eukaryote Transcriptome Sequencing Project (MMETSP): illuminating the functional diversity of eukaryotic life in the oceans through transcriptome sequencing. PLoS Biol. 2014, 12:e1001889.

29. Matasci N, Hung LH, Yan Z, Carpenter EJ, Wickett NJ, Mirarab S, Nguyen N, Warnow T, Ayyampalayam S, Barker M, et al.: Data access for the 1,000 Plants (1KP) project. GigaScience 2014, 3:17.

30. Kim S, Park MG: Paulinella longichromatophora sp. nov., a New Marine Photosynthetic Testate Amoeba Containing a Chromatophore. Protist 2016, 167:1-12.

31. Nowack EC, Price DC, Bhattacharya D, Singer A, Melkonian M, Grossman AR: Gene transfers from diverse bacteria compensate for reductive genome evolution in the chromatophore of Paulinella chromatophora. Proc. Natl. Acad. Sci. USA 2016, 113:12214-12219.

32. O'Neill EC, Trick M, Hill L, Rejzek M, Dusi RG, Hamilton CJ, Zimba PV, Henrissat B, Field RA: The transcriptome of Euglena gracilis reveals unexpected metabolic capabilities for carbohydrate and natural product biochemistry. Mol. Biosyst. 2015, 11:2808-2820.

33. Yoshida $Y$, Tomiyama T, Maruta T, Tomita M, Ishikawa T, Arakawa K: De novo assembly and comparative transcriptome analysis of Euglena gracilis in response to anaerobic conditions. BMC Genom. 2016, 17:182.

34. Wang WJ, Wang FJ, Sun XT, Liu FL, Liang ZR: Comparison of transcriptome under red and blue light culture of Saccharina japonica (Phaeophyceae). Planta 2013, 237:1123-1133.

35. Fortunato AE, Jaubert M, Enomoto G, Bouly JP, Raniello R, Thaler M, Malviya S, Bernardes JS, Rappaport F, Gentili B, et al.: Diatom Phytochromes Reveal the Existence of Far-Red-Light-Based Sensing in the Ocean. Plant Cell 2016, 28:616-628.

36. Hehenberger E, Burki F, Kolisko M, Keeling PJ: Functional Relationship between a Dinoflagellate Host and Its Diatom Endosymbiont. Mol. Biol. Evol. 2016, 33:2376-2390.

37. Campbell EL, Hagen KD, Chen R, Risser DD, Ferreira DP, Meeks JC: Genetic analysis reveals the identity of the photoreceptor for phototaxis in hormogonium filaments of Nostoc punctiforme. J. Bacteriol. 2015, 197:782791.

38. Savakis P, De Causmaecker S, Angerer V, Ruppert U, Anders K, Essen LO, Wilde A: Light-induced alteration of c-di-GMP level controls motility of Synechocystis sp. PCC 6803. Mol. Microbiol. 2012, 85:239-251. 
39. Gan F, Zhang S, Rockwell NC, Martin SS, Lagarias JC, Bryant DA: Extensive remodeling of a cyanobacterial photosynthetic apparatus in far-red light. Science 2014, 345:1312-1317.

40. Zhao C, Gan F, Shen G, Bryant DA: RfpA, RfpB, and $\mathbf{R f p C}$ are the Master Control Elements of Far-Red Light Photoacclimation (FaRLiP). Front. Microbiol. 2015, 6:1303.

41. Ho MY, Gan F, Shen G, Zhao C, Bryant DA: Far-red light photoacclimation (FaRLiP) in Synechococcus sp. PCC 7335: I. Regulation of FaRLiP gene expression. Photosynth. Res. 2016.

42. Ho MY, Shen G, Canniffe DP, Zhao C, Bryant DA: Light-dependent chlorophyll f synthase is a highly divergent paralog of PsbA of photosystem II. Science 2016, 353.

43. Gan F, Shen G, Bryant DA: Occurrence of Far-Red Light Photoacclimation (FaRLiP) in Diverse Cyanobacteria. Life 2014, 5:4-24.

44. Gan F, Bryant DA: Adaptive and acclimative responses of cyanobacteria to farred light. Environ. Microbiol. 2015, 17:3450-3465.

45. Forest KT: Vivid watercolor paintbox for eukaryotic algae. Proc. Natl. Acad. Sci. USA 2014, 111:5448-5449.

46. Li FW, Villarreal JC, Kelly S, Rothfels CJ, Melkonian M, Frangedakis E, Ruhsam M, Sigel EM, Der JP, Pittermann J, et al.: Horizontal transfer of an adaptive chimeric photoreceptor from bryophytes to ferns. Proc. Natl. Acad. Sci. USA 2014, 111:6672-6677.

47. Bolige A, Goto K: High irradiance responses involving photoreversible multiple photoreceptors as related to photoperiodic induction of cell division in Euglena. J. Photochem. Photobiol. B. 2007, 86:109-120.

48. Suetsugu N, Mittmann F, Wagner G, Hughes J, Wada M: A chimeric photoreceptor gene, NEOCHROME, has arisen twice during plant evolution. Proc. Natl. Acad. Sci. USA 2005, 102:13705-13709.

49. Ermert AL, Mailliet K, Hughes J: Holophytochrome-Interacting Proteins in Physcomitrella: Putative Actors in Phytochrome Cytoplasmic Signaling. Front. Plant Sci. 2016, 7:613.

50. Chen M, Chory J: Phytochrome signaling mechanisms and the control of plant development. Trends Cell Biol. 2011, 21:664-671.

Figure Legends

Figure 1: Phytochrome domain architecture and photoisomerization. Representative domain architectures are shown for phytochromes with histidine kinase output domains from different lineages. Bilin is shown as a blue polygon. Protein domains are shown as cartoon shapes. Domain names are as follows: PAS, Per/ARNT/Sim domain; GAF, 
cGMP-specific phosphodiesterases/cyanobacterial adenylate cyclases/FhlA domain; PHY, phytochrome-specific domain (tongue shown in pink); His kinase, histidine kinase bidomain; S/T kinase, Ser/Thr kinase domain; REC, response regulator receiver domain; cyclase, adenylate cyclase domain; RING, really interesting new gene domain.

Figure 2: Phytochrome photoconversion. Absorption spectra are shown for plant phytochrome in the indicated photostates, with photoconversion and thermal recovery (dark reversion) of $\mathrm{P}_{\mathrm{fr}}$ shown.

Figure 3: Distribution of phytochrome in algae. A simplified view of the tree of life is shown, incorporating recent research on the origins of eukaryotes and on the evolution of Cryptista, stramenopiles, and dinoflagellates. Archaeplastida are shown as monophyletic for simplicity. Primary (dashed blue lines), secondary (thin solid lines), and tertiary (thick grey lines) endosymbioses are shown. Oxygenic photosynthetic organisms are colorcoded by light-harvesting strategy and plastid ancestry. 


\section{Figure 1}

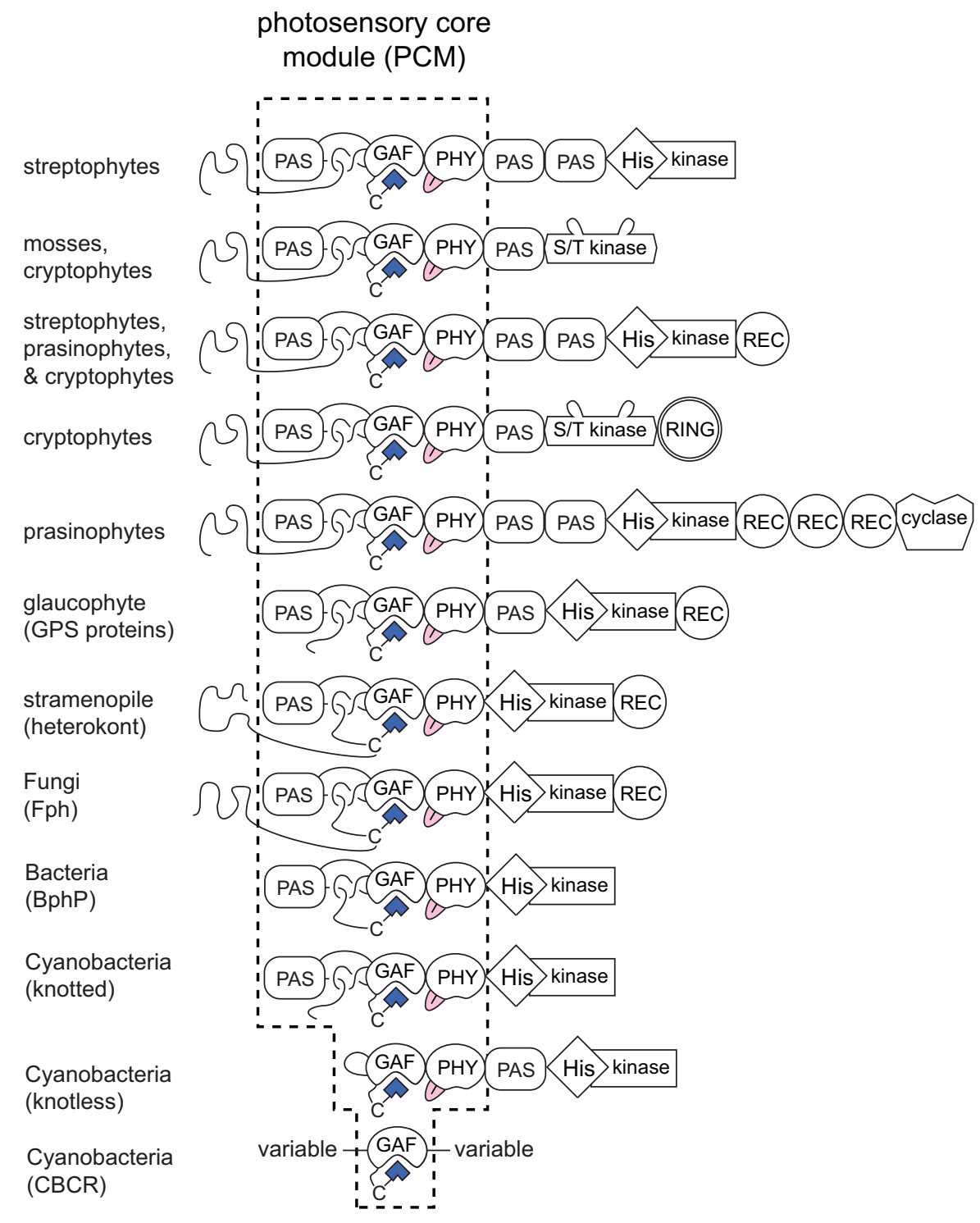




\section{Figure 2}
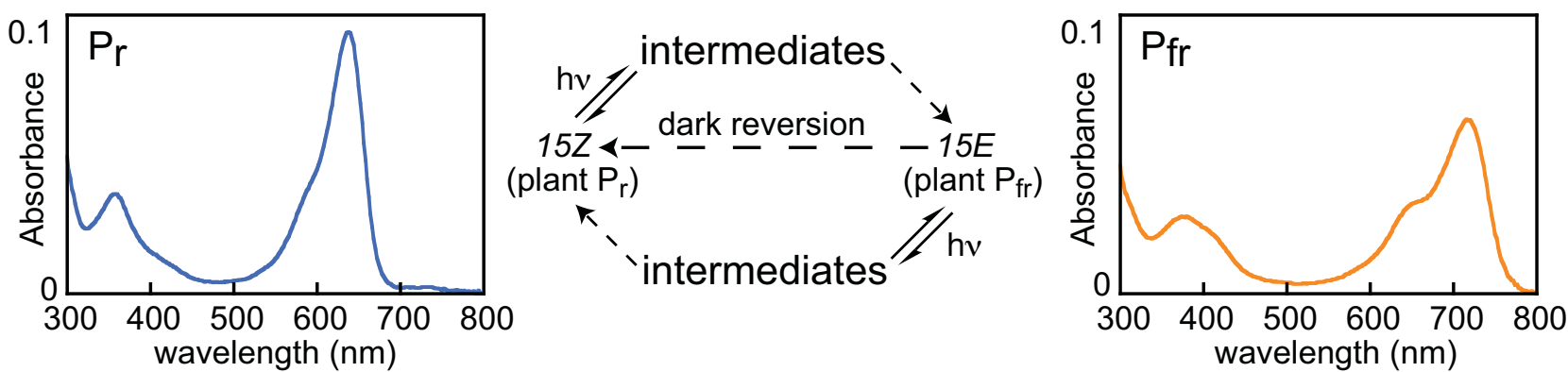


\section{Figure 3}

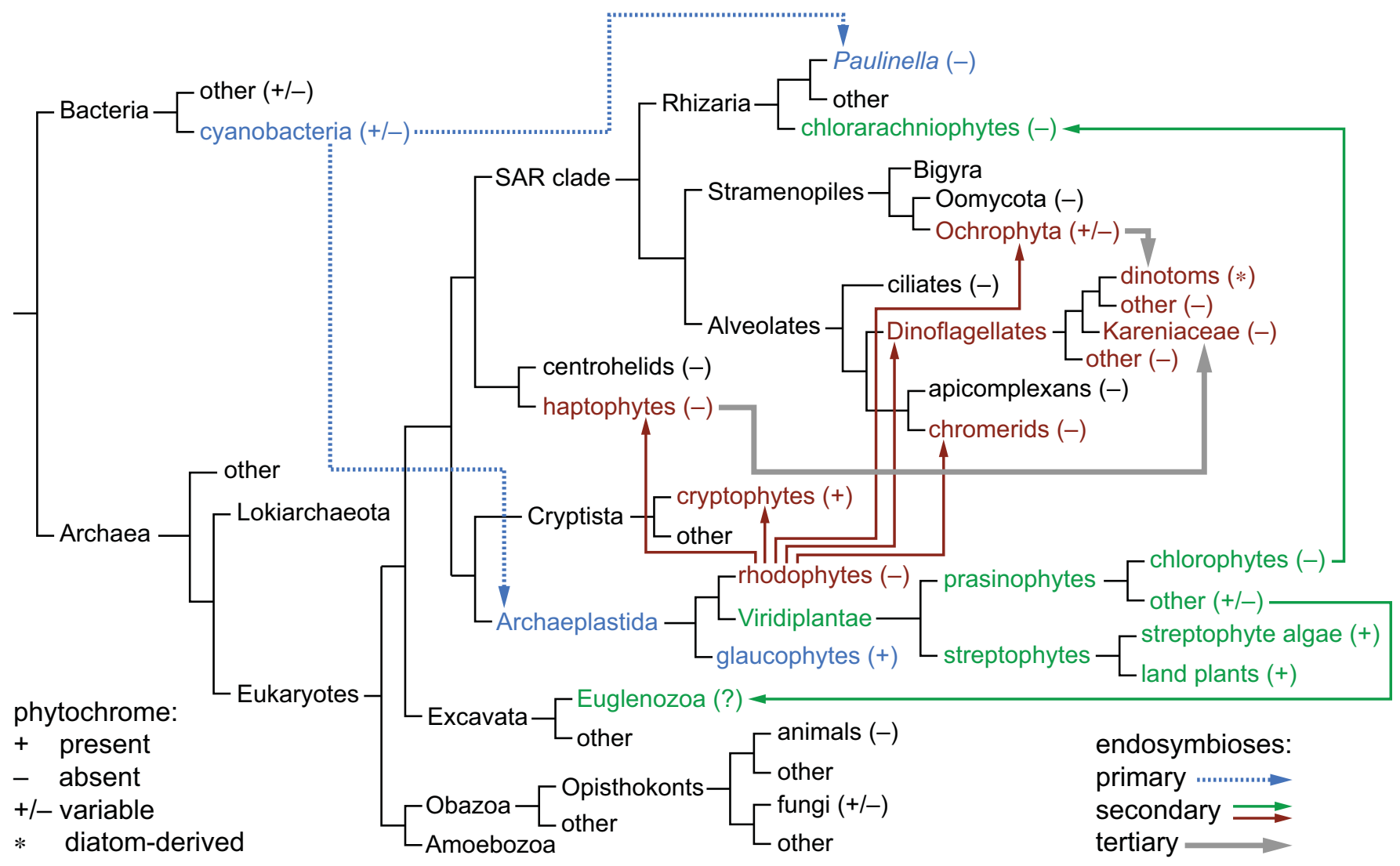

